\title{
AutoPas: Auto-Tuning for Particle Simulations
}

Fabio Gratl, Steffen Seckler, Nikola Tchipev, Hans-Joachim Bungartz Technical University of Munich

Faculty of Informatics

Chair of Scientific Computing in Computer Science (SCCS)

Rio de Janeiro, May 24. 2019

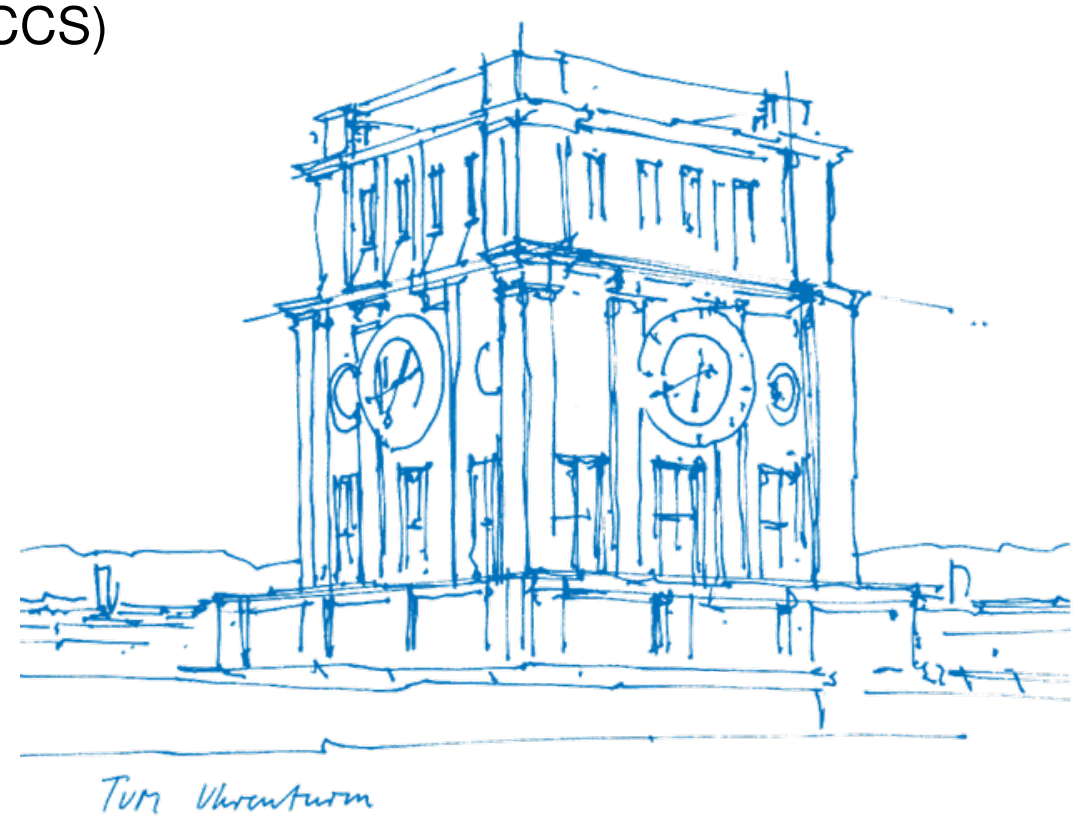




\section{Motivation}

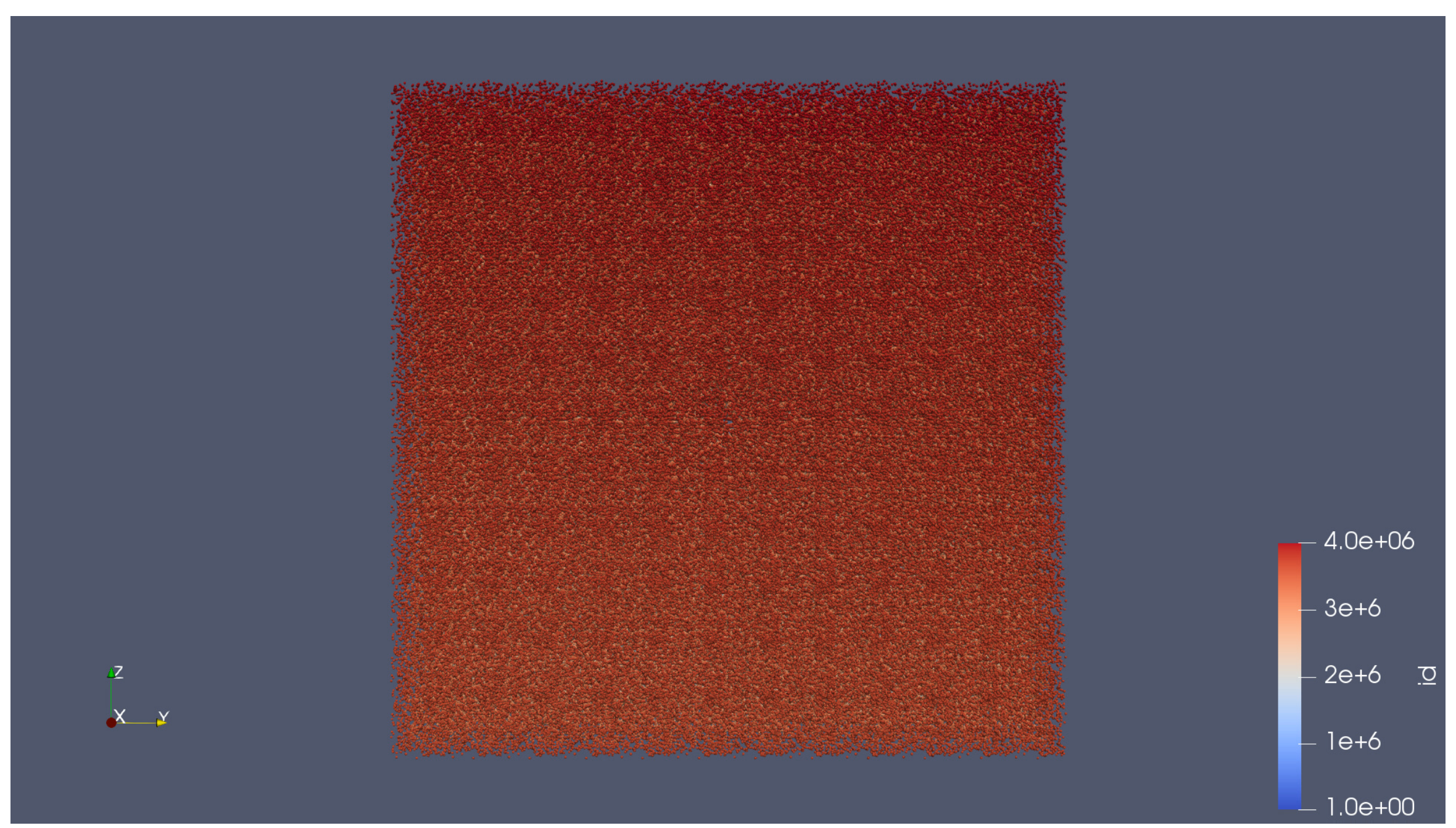




\section{Outline}

\section{Molecular Dynamics}

\section{Basics}

Challanges

\section{AutoPas}

Implemented Algorithms

Auto-Tuning

Integration

\section{Experimental Results}




\section{Molecular Dynamics}




\section{Molecular Dynamics}

- Here: small rigid molecules

- Simulation of movement of molecules

- Computation of pairwise forces

- Newtons Laws of Motion

- $N$-Body problem $\Rightarrow O\left(N^{2}\right)$

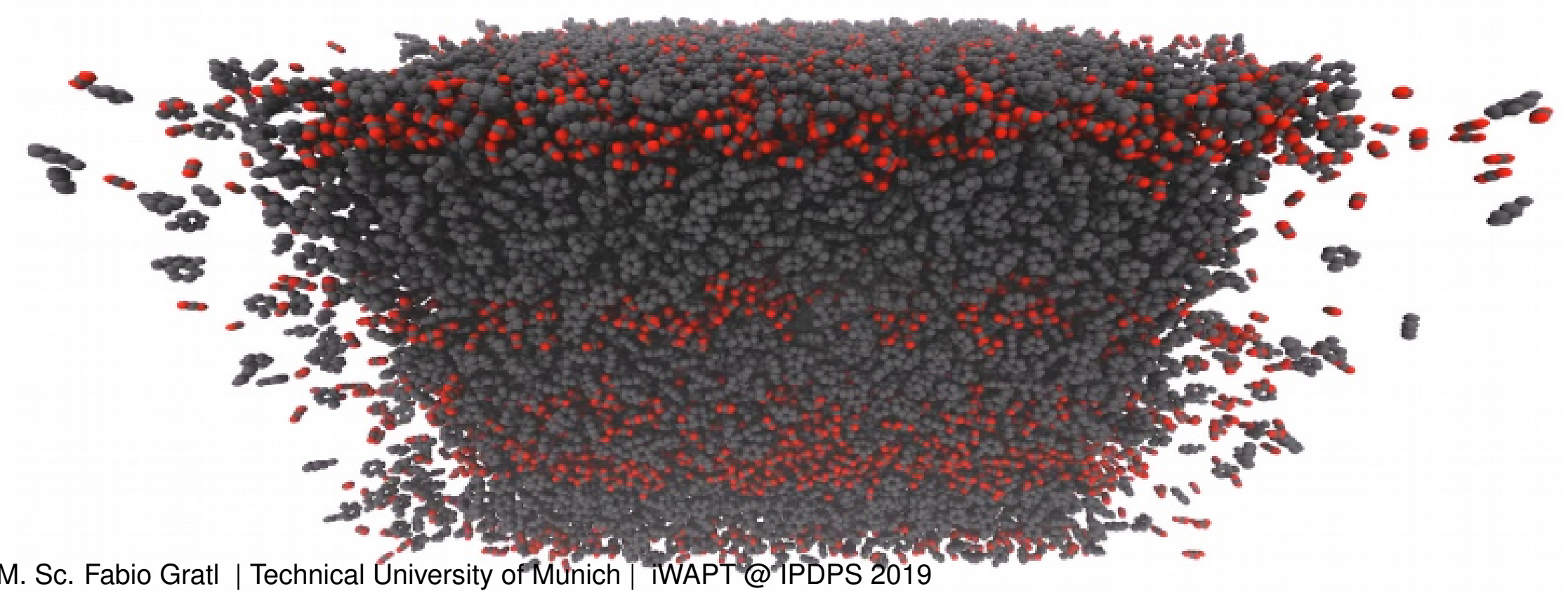




\section{Short Range Interactions: Lennard-Jones Potential}

$$
U\left(x_{i}, x_{j}\right)=4 \varepsilon\left(\left(\frac{\sigma}{\left\|x_{i}-x_{j}\right\|_{2}}\right)^{12}-\left(\frac{\sigma}{\left\|x_{i}-x_{j}\right\|_{2}}\right)^{6}\right)
$$

- Characteristics of molecule type:

$-\varepsilon$ : Potential well

$-\sigma:$ Zero crossing

- Cutoff $r_{c}$

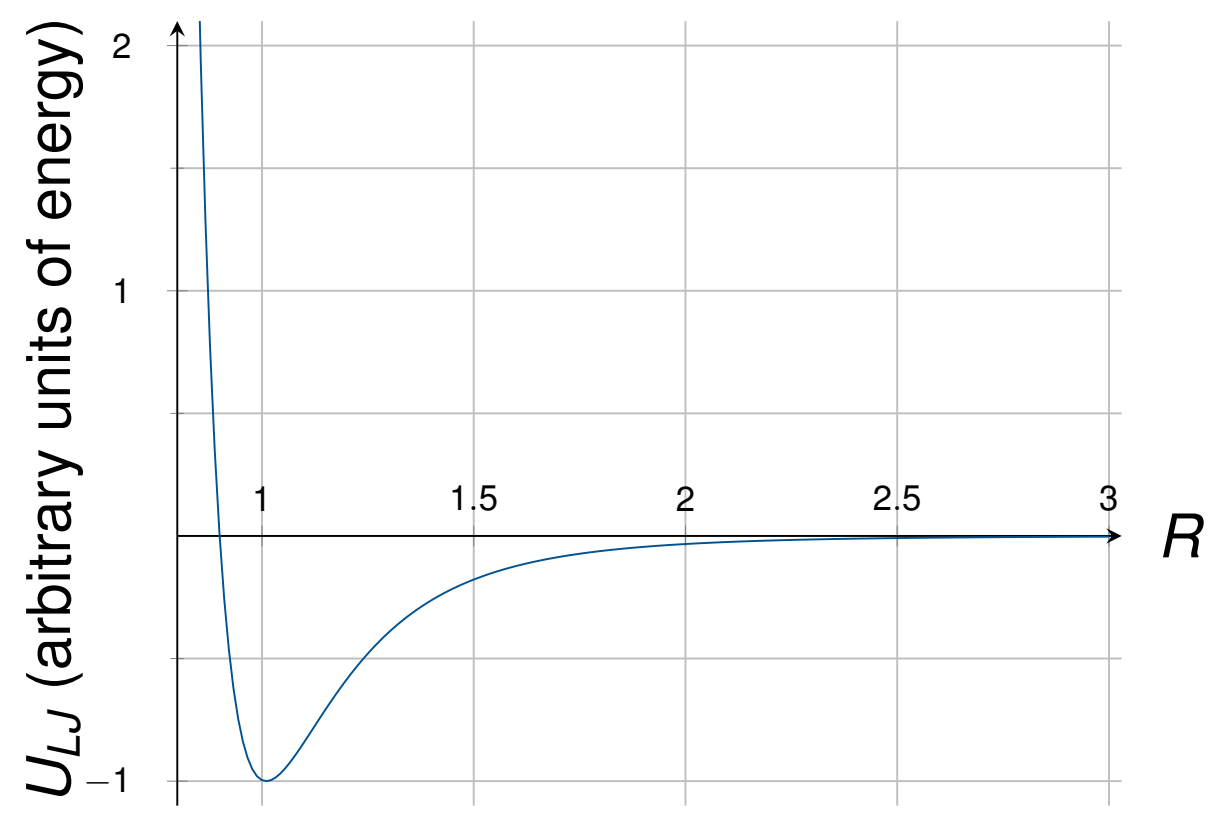

Figure: LJ-Potential for $\varepsilon=1$ and $\sigma=0.9$ 


\section{Challanges}

- Total number of particles

- Particle density

- (In-)Homogeneity

- Systems changing over time

- Many possible algorithms

- Overall goal: Minimize time to solution!

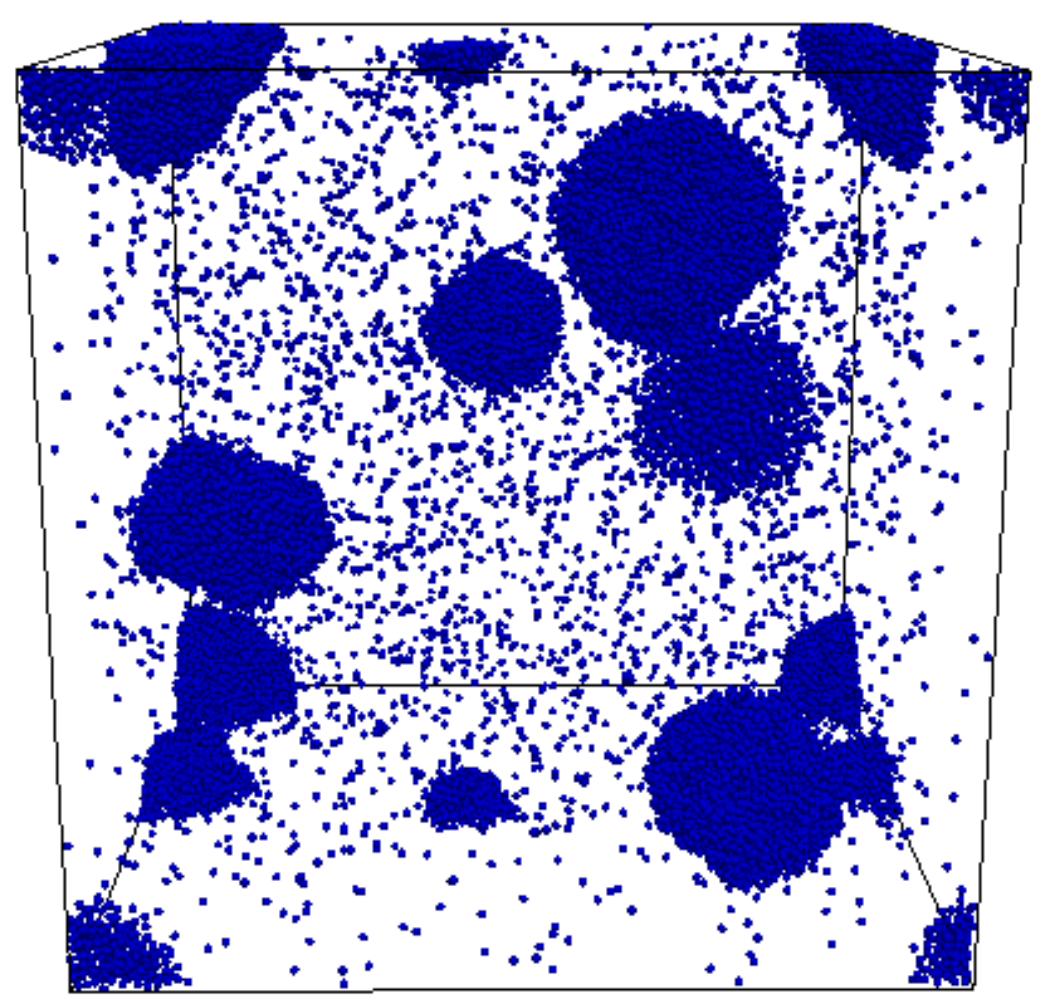




\section{AutoPas}




\section{AutoPas: Overview}

- Node-Level C++ header library

- User defines:

- Properties of particles

- Force for pairwise interaction

- AutoPas provides:

- Containers, Traversals, Data Layouts, ...

- Dynamic Tuning at run-time

- Black Box container

$\Rightarrow$ General base for $N$-Body simulations

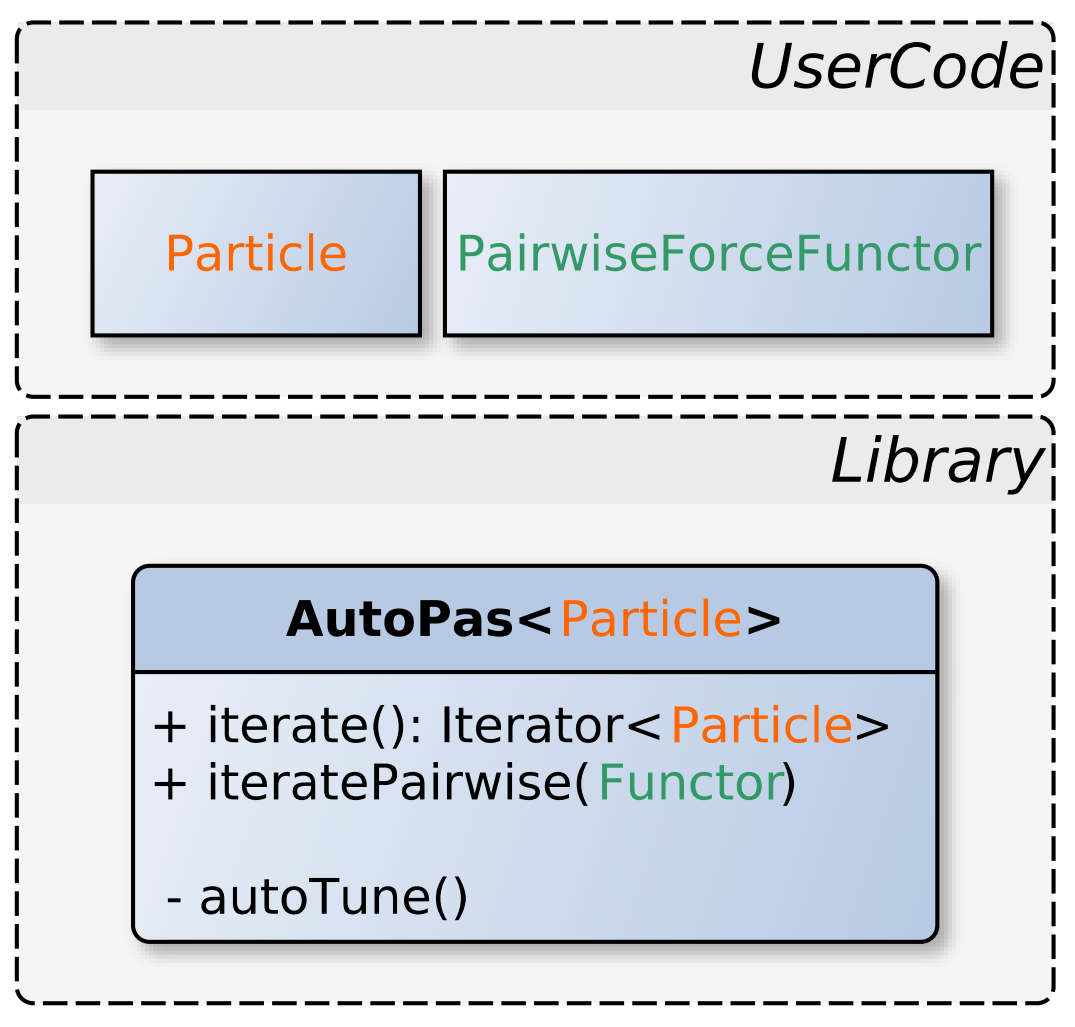

https://github.com/AutoPas/AutoPas 


\section{Container Options}

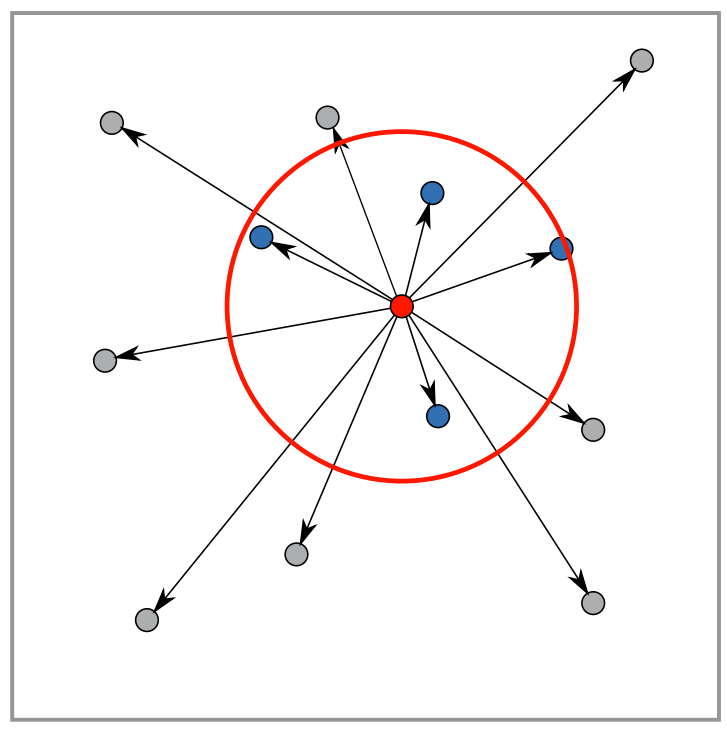

Direct Sum

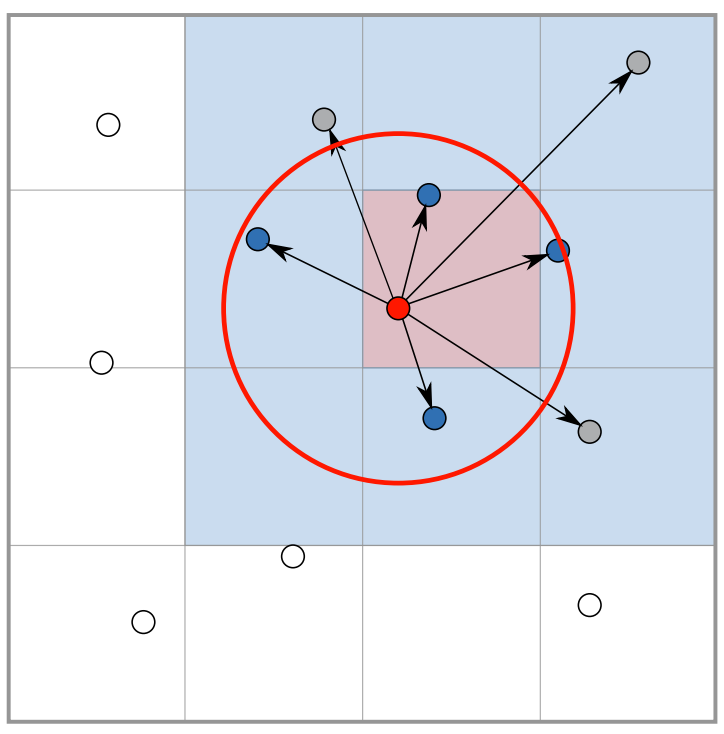

Linked Cells

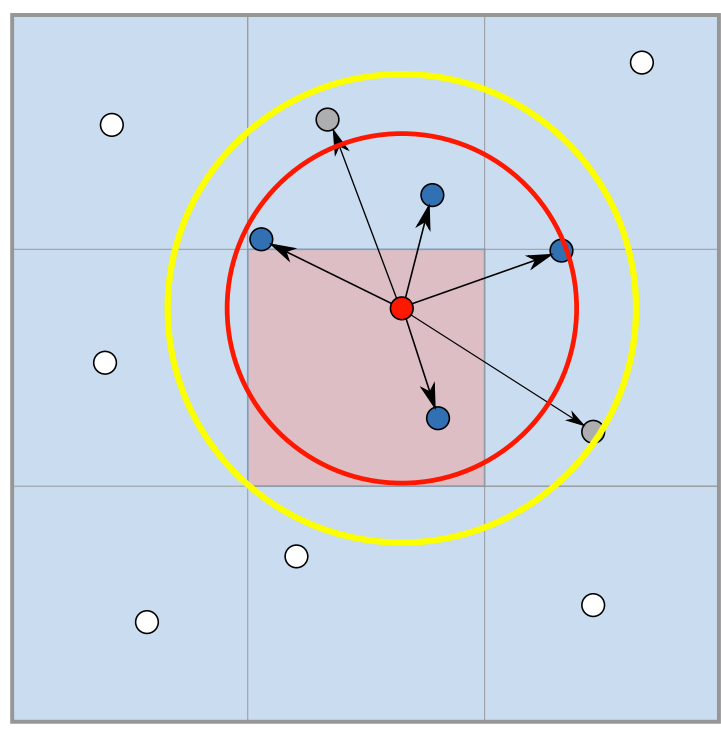

Verlet Lists

Memory Overhead

\section{Computational Overhead}




\section{Container Comparison}

- Every container has advantages

- Linked Cell benefits most from SoA

$\Rightarrow$ best in dense scenarios

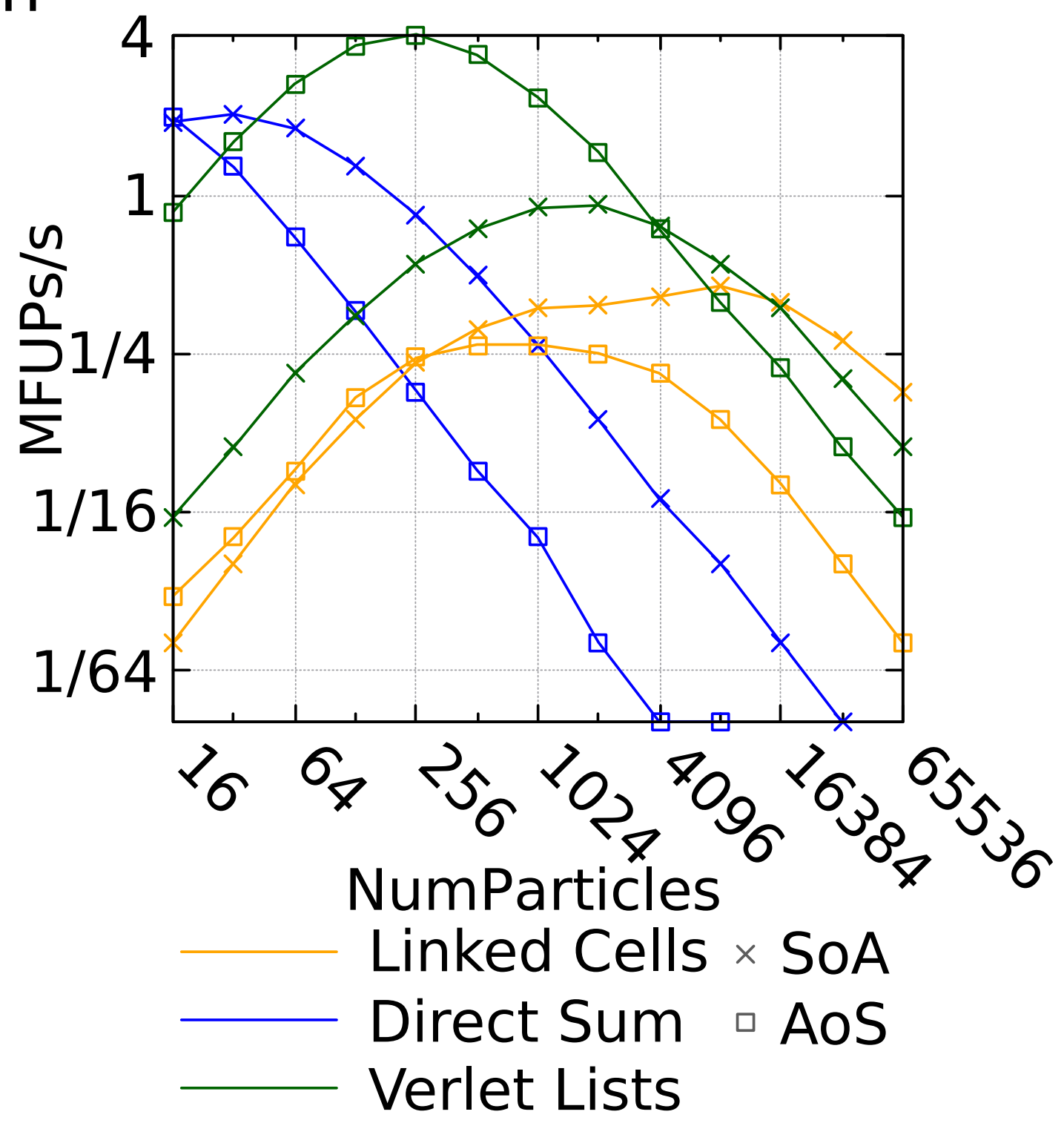




\section{Linked Cells Parallelization Options}

\begin{tabular}{|l|l|l|l|l|l|l|l|l|}
\hline 56 & & 63 & 56 & & & 59 & 60 & \\
\hline \\
\hline 48
\end{tabular}




\section{Hardware Comparison / Threads}

- Traversals: c08: 8-way domain coloring sliced: regular 1D domain partitioning

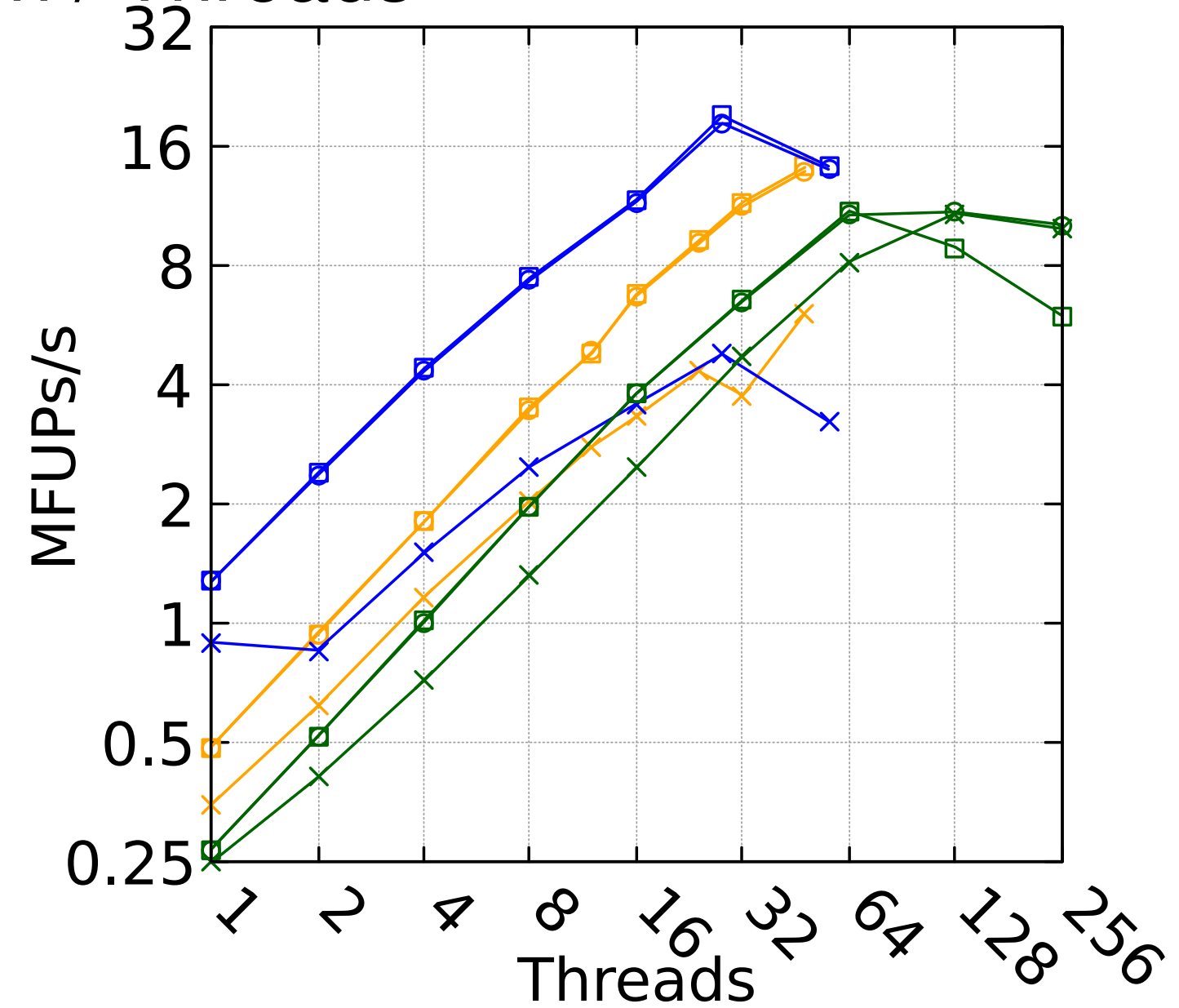

Magny

- Haswell KNL $\times$ c08

$\square$ sliced

- tune 


\section{Hardware Comparison / Data Layout}

- Vector Instructions: \begin{tabular}{l|l|l} 
Magny & SSE4 & 128
\end{tabular} Haswell AVX2 256 KNL AVX512 512

$\Rightarrow$ Optimal data layout dependent on hardware

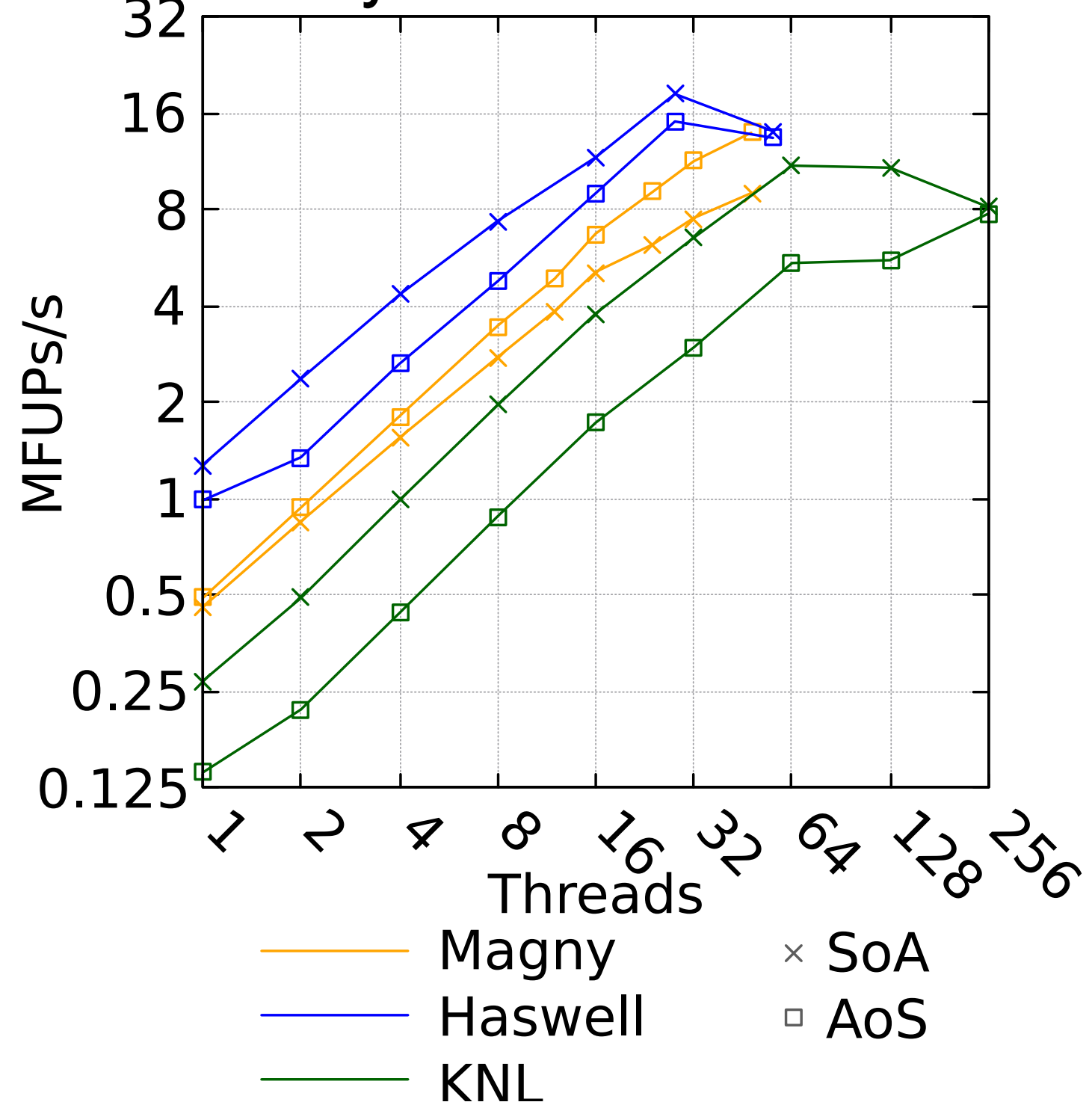




\section{Auto-Tuning Process}

- Common interfaces for containers, traversals, etc

$\Rightarrow$ Strategy pattern

- Repeated periodically

- User can restrict testing space

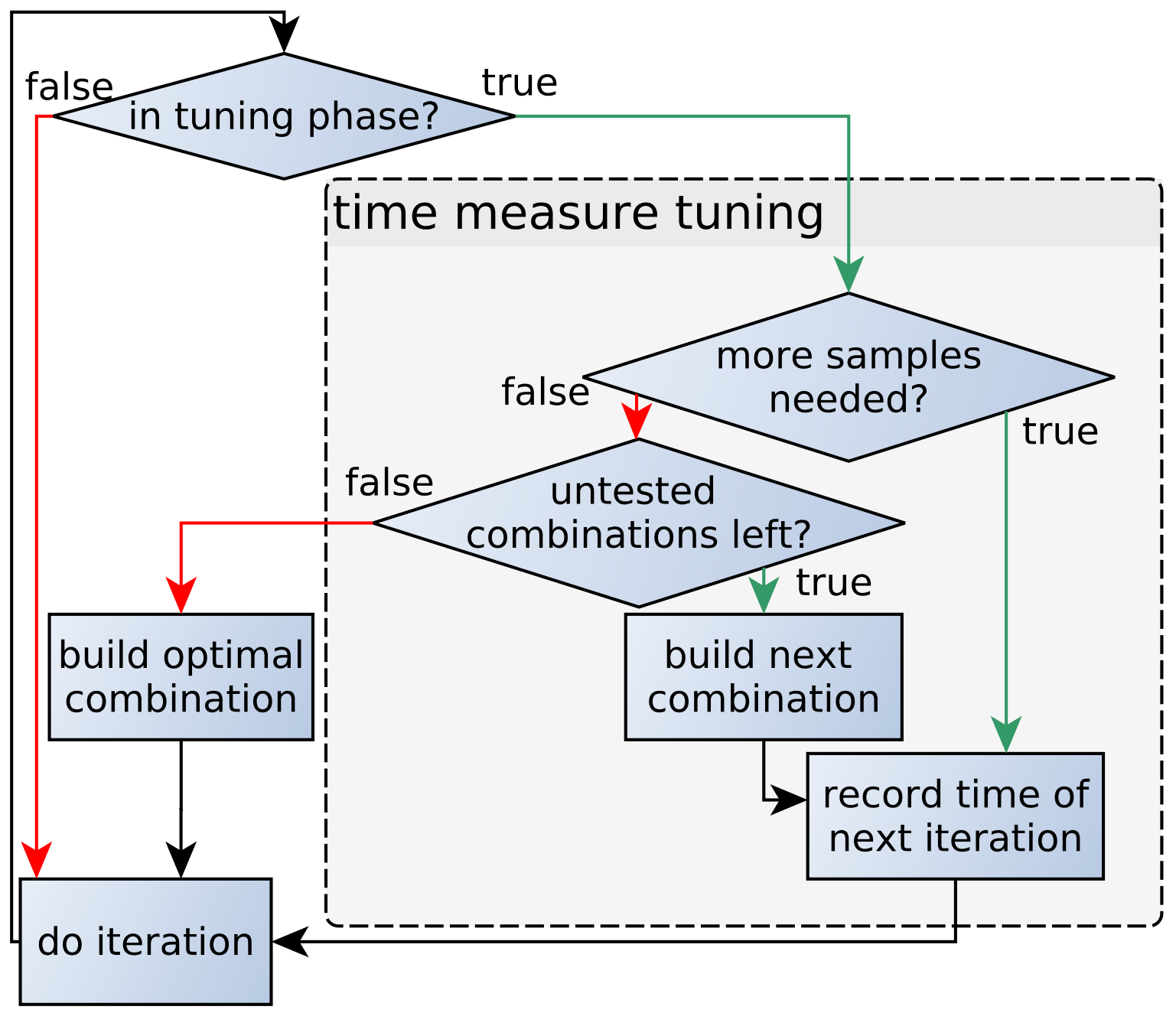




\section{Integration into Is1 mardyn}

- Is1 mardyn:

- Large number of small rigid molecules.

- Actively used in chemical engineering.

- Example Lennard-Jones functor from AutoPas

- New particle class

- Inherits from AutoPas and Is1 mardyn particle interface.

- Acts as coupler

- New particle container class

- Only wrapper around AutoPas main interface.
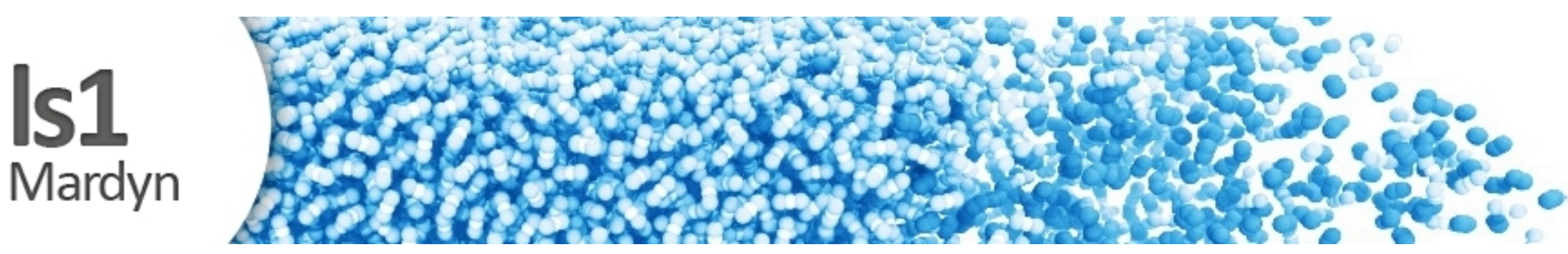


\section{Experimental Results}




\section{Scenario: Spinodal Decomposition}

- 4008960 particles

- Block size: $240 \times 240 \times 240$

- Periodic boundary conditions

- Cutoff = 2.5

- Sub-critical temperature

- Rapid and drastic change in homogeneity

$\Rightarrow$ Interesting target for tuning!
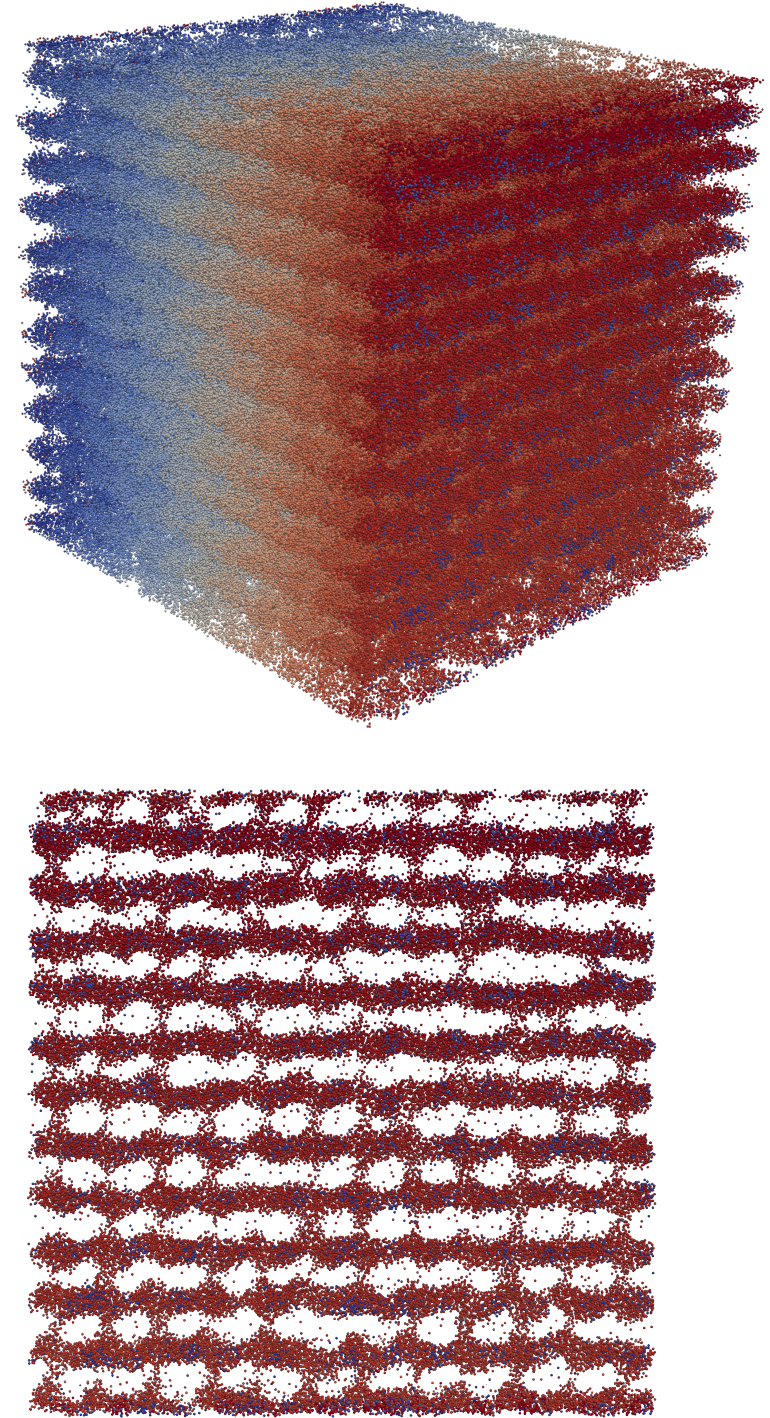


\section{Tuning Behavior}

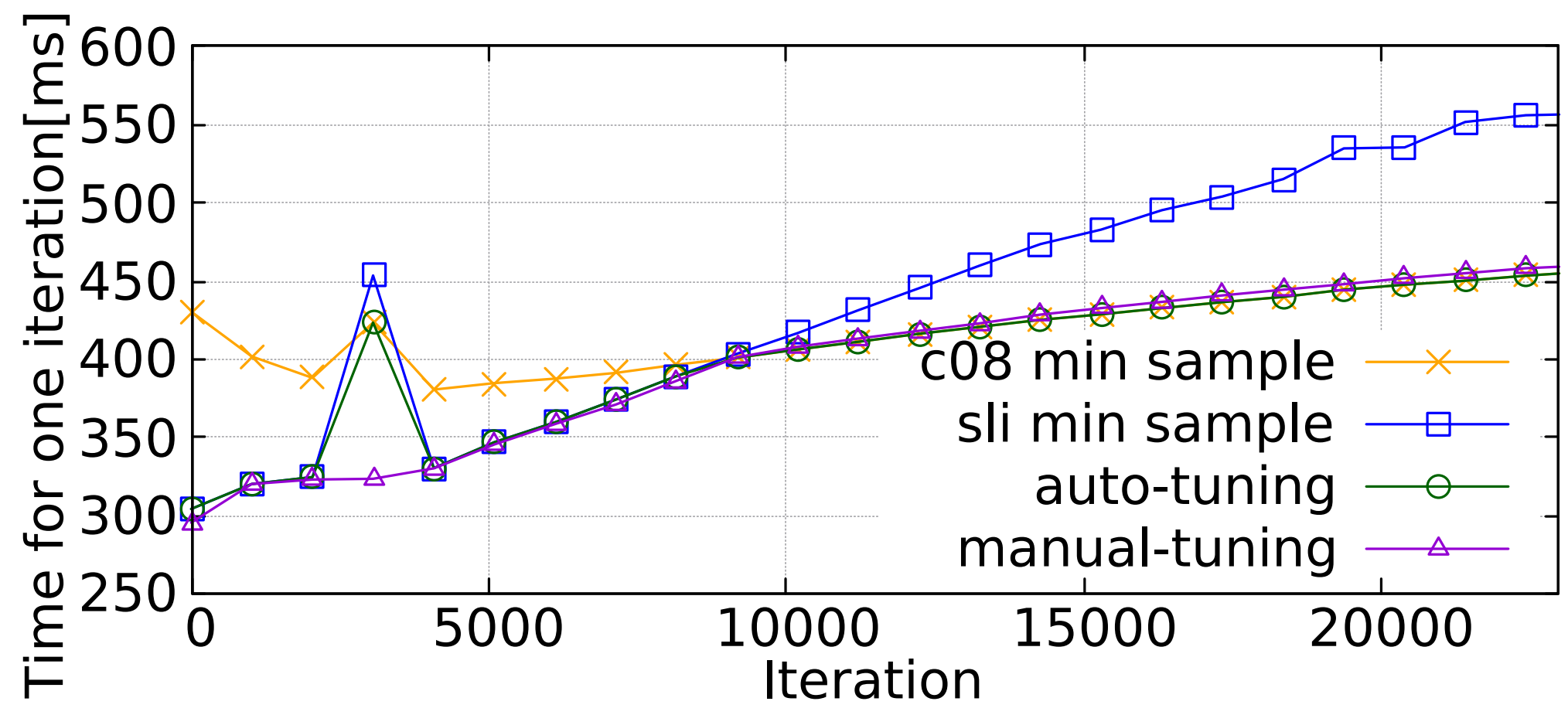

- Tuning switches as expected

- Misclassification can happen 


\section{Tuning Overhead}

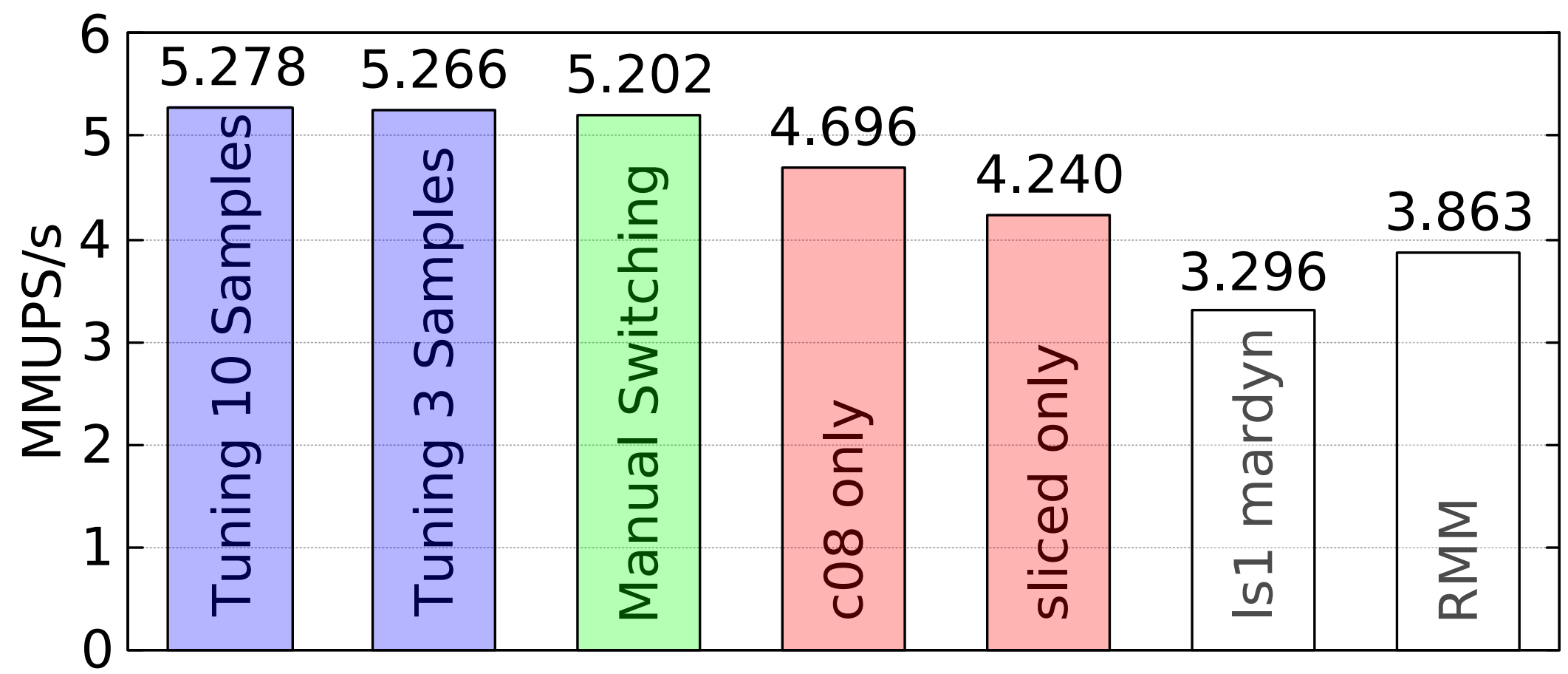

- Tuning and manual switching equally fast. $\Rightarrow$ No overhead from tuning!

- Tuning faster than static configuration.

- Faster than original Is1 mardyn, even in Reduced Memory Mode. 


\section{Conclusions}

- AutoPas is a black box $N$-Body container.

- Dynamic tuning enables optimal performance for changing scenarios.

- Achievable for users without expert knowledge.

- Easy to integrate in existing codes.

... and future work:

- AutoPas + MPI.

- More / optimized algorithms.

- Tuning for more parameters.

- Search space reduction. 